\title{
ON THE DIFFERENTIATION OF STREPTOMYCIN AND ALLIED SUBSTANCES (STREPTOTHRICIN GROUP) AND RAPID ISOLATION OF STREPTOMYCIN.PRODUCING STRAIN
}

\author{
Hamao UMEZAWA, SeIKI HAYANO and Yasuo OGATA \\ National Institute of Health of Japan, Tokyo
}

Since we undertook the studies on streptomycin, various kinds of actinomyces producing substances resembling streptomycin were isolated $(1,2)$, and we were thrown into confusion, as to which one of them was streptomycin. The difficulties of the isolation of streptomycin-producing strain seem to be ascribable to the existence of many kinds of actinomyces producing other substances resembling streptomycin. which, in the broth, inhibit B. coli and other gram negative bacteria rather more strongly than streptomycin.

Streptomycin, streptothricin (3) and allied substances are usually differentiated on the basis of their antibacterial spectra, but this differentiation method can only be utilized in laboratories, where streptomycin, streptothricin and other related substances are already isolated, since even among the same species the resistance of bacteria may be different in different individual strains.

Recently Waksman, Reilly, and Jonstone (4) reported that' streptomycin-producing strain was much more resistant against streptomycin and the incorporation of streptomycin in the isolating medium in concentration sufficient to inhibit the growth of the usual actinomyces facilitated the isolation of streptomycin strain. Among 120 antibiotic strains of various kinds of actinomyces isolated by us, 21 strains grew on the nutrient agar plus $50 \mu \mathrm{g}$ of streptomycin hydrochloride (Merck's sample, about $600 \mu / \mathrm{mg}$ ) and from one of them streptomycin was extracted and purified as its reineckate crystal. This and other strains producing related substances and the antibiotic substances extracted from them were comparatively studied, and it was concluded that among these substances streptomycin was particular in respect of the tolerance of streptomycin-fast bacteria and the colour of its Sakaguchi's reaction, though they were all similar in extractability and solubility, and inhibited both gram positive and negative bacteria.

Now we should like to devide the antibiotic substances resembling streptomycin and streptothricin into streptomycin and streptothricin-group substances, among the latter streptothricin having been first isolated by Waksman and others (3). Lavendulin and actinorubin found by Kelner and Morton (5) and basic antibiotic substances resembling streptomycin and streptothricin reported by us $(1,2)$ are to be included in streptothricingroup substances, for the streptothricin-fast B. coli is nearly as susceptible to these substances as the normal culture. Streptin found by Foster and others (6) also seems to belong to the streptothricin-group substances, for it is extracted in the same way as streptomycin from the broth of A. reticulus-ruber, which was found by us to produce a streptothricin group substances and a streptomycin-producing strain is not yet found in 
species other than S. griseus.

On account of the possible differentiation of streptomycin from allied substances and also of the important discovery by Waksman and others (4), showing the specific tolerance of streptomycin-producing strain against streptomycin, the isolation of the streptomycin strain became much easier.

In the present paper method for the differentiation of streptomycin and strepthothricingroup substances and the rapid isolation and identification of streptomycin-producing strain are described.

\section{Development of tolerance of E. coli to streptomycin and streptothricin-group substances}

Basic antibiotic substances resembling streptomycin and streptothricin were extracted in our laboratory from A. albus (No. 69 and No. 284), A. lavendulae (No. 201), A. ruber (No. 506), A. reticulus-ruber (No. 9), A. microflavus (No. 13A), and No. E-2 strain, and recently streptomycin was extracted from a strain of $\mathrm{S}$. griseus growing well on the nutrient agar plus streptomycin.

In the course of serial transfers successively on the nutrient agar added with these antibiotic substances, $\mathrm{E}$. coli became more resistant against the added substance than the normal strain, and the tolerance of $\mathrm{E}$. coli to streptomycin increased much more rapidly than in the case of other substances. Then a strain of E. coli the resistance of which was increased against one of these substances was tested to see whether it became resistant against other substances also. In this experiment, the tolerance was tested by serial dilution of the antibiotic substances in the broth, finding the maximum dilution sufficient to inhibit completely the growth of the organism. From the broth employed in this test sodium chloride was removed and $\mathrm{pH}$ was adjusted to 7.5 , as the bacteriostatic effect of these substances was hindered by sodium chloride, and became stronger in the

Table 1. The maximum dilutions of streptomycin and streptothricin-group substances sufficient to inhibit completely a streptomycin-fast strain of E. coli and several strains of E. coli resistant to streptothricin-group substances

\begin{tabular}{l|r|r|r|r|r|r|r}
\multicolumn{1}{c|}{ E. coli resistant to } & \multicolumn{5}{c|}{$\begin{array}{c}\text { Streptothricin-group represented } \\
\text { by strain number }\end{array}$} & Streptomycin \\
\cline { 2 - 8 } & 9 & E-2 & $13 \mathrm{~A}$ & 284 & 201 & 69 & $\mathrm{~S}-1$ \\
\hline The normal culture & 128 & 128 & 16 & 8 & 32 & 10 & 64 \\
to streptomycin & 256 & 128 & 12 & 32 & 32 & 10 & $<0.5$ \\
to No. 9 & 4 & 32 & 0.5 & 0.5 & 1 & 0 & $<0.5$ \\
to E-2 & 32 & 16 & 0.5 & 4 & - & 2 & 4 \\
to 13A & - & - & 0.5 & - & 2 & 1.2 & 2
\end{tabular}

(1) 9: A. reticulus-ruber
284: A. albus
201: A. lavendulae
69: A. albus
13A: A. microflavus
S-1: A. griseus.

(2) figures $\times 1,000$ present the maximum dilution. 
more alkaline broth. As shown in Table 1, the streptomycin-fast strain is nearly as susceptible to other substances as the normal strain, but the strain which became resistant to one of the streptothricin-group substances is not only resistant to all other streptothricin-group substances but also to streptomycin.

\section{Bacteriostatic spectra of streptomycin and streptothricin-group substances}

A round filter paper with the diameter of $5 \mathrm{~mm}$, was moistened with water solution of streptomycin or streptothricin-group substances and put on the centre of the nutrient agar plate $(\mathrm{pH} 8.0)$. After the plate had been preserved for eighteen hours at $5^{\circ} \mathrm{C}$ in order to permit these substances to diffuse, various kinds of bacteria were streaked radially from the filter paper, and after 20 hours at $37^{\circ} \mathrm{C}$, the length of the inhibition zones was measured. The results are shown in Table 2.

Table 2. The length $(\mathrm{mm})$ of the inhibition zones from the edge of the filter paper observed in various kinds of bacteria

\begin{tabular}{|c|c|c|c|c|c|c|c|c|}
\hline \multirow{2}{*}{ Bacterial species } & \multicolumn{7}{|c|}{$\begin{array}{l}\text { Streptothricin-group represented } \\
\text { by strain number }\end{array}$} & \multirow{2}{*}{$\begin{array}{l}\text { Strepto- } \\
\text { mycin }\end{array}$} \\
\hline & $\mathrm{E}-2$ & 9 & 284 & 69 & 201 & $13 \mathrm{~A}$ & 506 & \\
\hline E. coli & 11.0 & 11.2 & 11.5 & 7.6 & 6.5 & 7.3 & 5.2 & 13.3 \\
\hline $\begin{array}{l}\text { Streptomycin-fast E. coli } \\
\text { S. aureus }\end{array}$ & 9.5 & 10.5 & 10.5 & 6.1 & 4.9 & 5.8 & 2.1 & 0 \\
\hline Heatley strain & 10.4 & 10.6 & 10.7 & 7.5 & 6.4 & 6.5 & 4.3 & 13.7 \\
\hline $209-\mathrm{p} \quad$ & 11.4 & 12.0 & 12.0 & 10.6 & 7.7 & 7.6 & 6.2 & 13.9 \\
\hline Terajima " & 12.1 & 12.1 & 12.4 & 10.6 & 7.1 & 7.6 & - & 14.2 \\
\hline S. dysenteriae & 10.0 & 10.4 & 11.2 & 6.5 & 5.4 & - & - & 12.3 \\
\hline S. typhi-murium . & 10.2 & 10.8 & 10.5 & 6.9 & 5.6 & 5.9 & - & 10.6 \\
\hline E. typhi & 9.2 & 9.4 & - & 6.5 & 5.8 & - & - & 10.9 \\
\hline B. melitensis & 10.9 & 9.9 & 10.7 & 7.3 & 5.7 & - & - & 12.0 \\
\hline $\begin{array}{l}\text { B. subtilis (a) } \\
\text { (N. R. R. L. B-558) }\end{array}$ & 11.0 & 10.5 & 11.6 & 7.5 & 6.8 & 7.2 & 5.0 & 7.6 \\
\hline B. anthracis (b) & 7.8 & 7.6 & 7.4 & 3.7 & 3.2 & 3.7 & 3.2 & 15.7 \\
\hline$a-b$ & 3.2 & 2.9 & 4.2 & 2.8 & 36 & 3.5 & 2.8 & -8.1 \\
\hline
\end{tabular}

The streptomycin-fast $\mathrm{E}$. coli is much more resistant to streptomycin than the normal culture, but only very slightly more resistant to other substances. No marked difference was observed in the resistances among S. aureus, E. typhi, S. typhi-murium, Shigella dysenteriae, B. melitensis to streptomycin and streptothricin-group substances. But in the case of B. subtilis N.R.R. L. B-558 and B. anthracis there is a marked difference. Against streptomycin B. subtilis was much more" resistant than B. anthracis, but against streptothricin-group substances the former was more susceptible than the latter. In this experiment no marked difference was observed among streptothricin-group substances. 


\section{Other differences bctween streptomycin and streptothricin-group substances}

Both streptomycin and streptothricin-group substances can be adsorbed by active carbon from the culture liquid ( $1 \%$ glucose bouillon), then eluted into acid methanol and

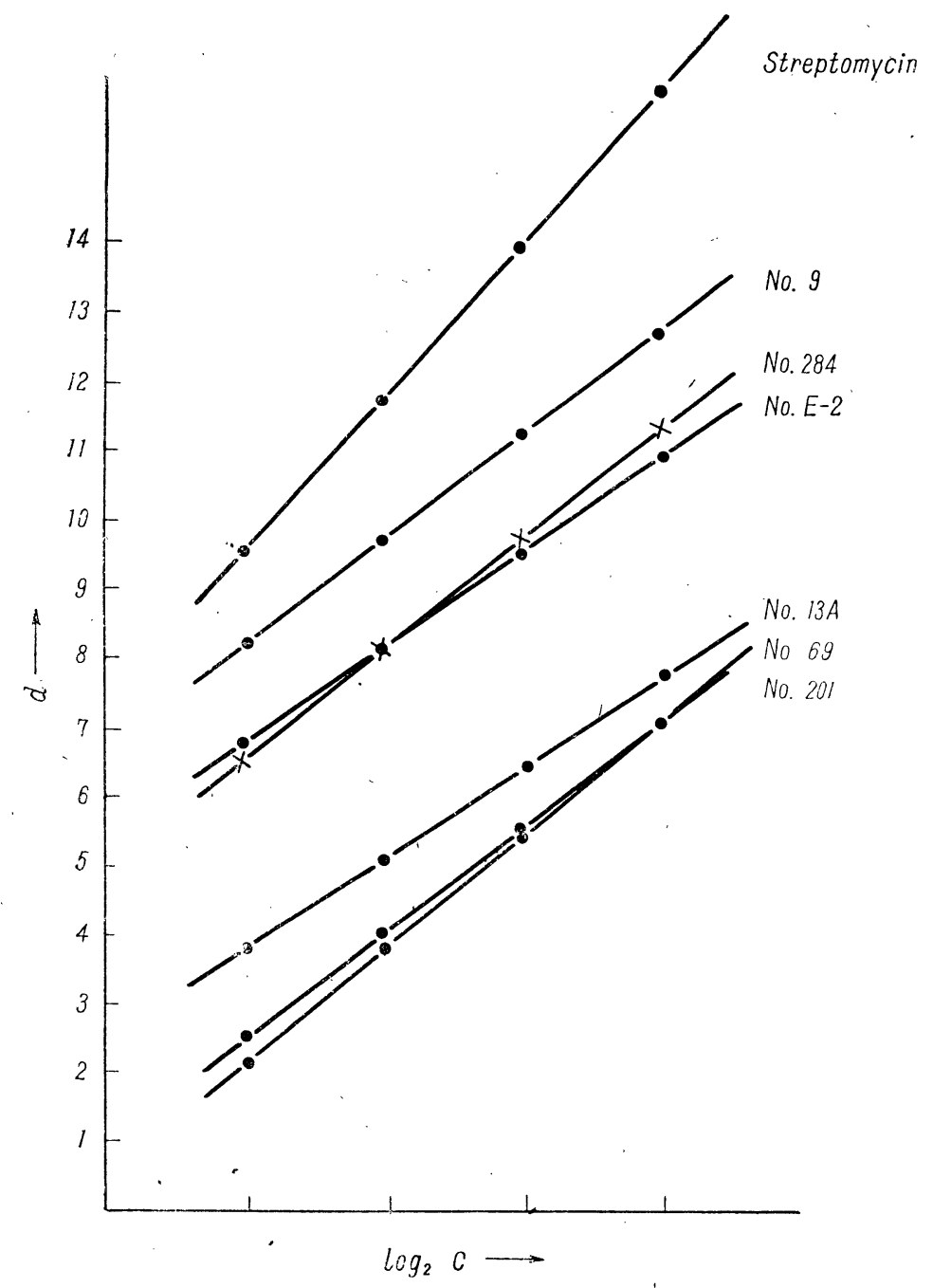

Fig. 1. The relation, $d=\alpha+\beta \log c$, observed.

$\mathrm{d}=$ diameter of inhibition zone

$c=$ concentration of the antibiotic substances.

$\alpha, \beta=$ constants

precipitated by ether as their hydrochlorides. In this extraction stage we tested the Sakaguchi reaction of these substances, and found strepromycin hydrochloride to present beautiful red colour, but hydrochlorides of streptothricin-group substances only brownish red colour. In this extraction stage these substances contain a lot of impurities, so that 
this colour of streptothricin-grqup substance may be due to impurities, but this difference seems to be utilizable to decide whether the extracted substance is stréptomycin or not.

If the relations between the concentrations (c) and diameters (d) of inhibition zones are tested by paper disc method, the relation, $d=\alpha+\beta \log c$ ( $\alpha$ and $\beta$ are constants), can be observed in all these substances. But, as shown in Fig. $1, \beta$ observed in the case of streptomycin is much larger than those in the case of streptothricin-group substances.

In addition to these differences between streptomycin and streptothricin-group substances already described, another important difference was also observed. As Waksman (3) noticed from the beginning of his studies, streptothricin-group substances showed the lately appearing toxicity. By the intravenous injection of minimal lethal dose of streptomycin mice died in 24 hours, but in the case of streptothricin-group substances (No. 284, No. 9, No. 13A, No. E-2) mice died irregularly after 48 hours.

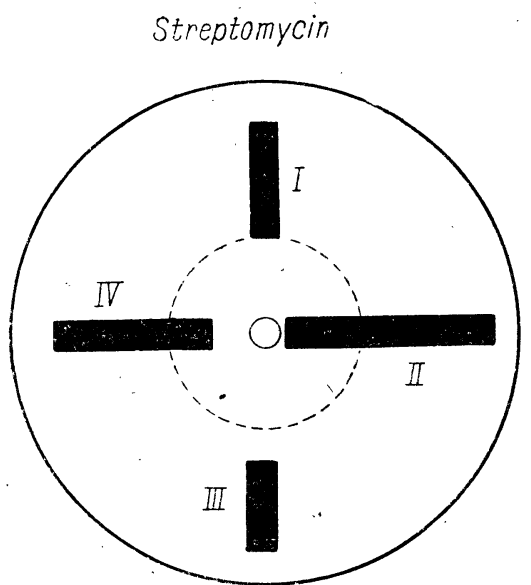

\section{Streptothricin-group}

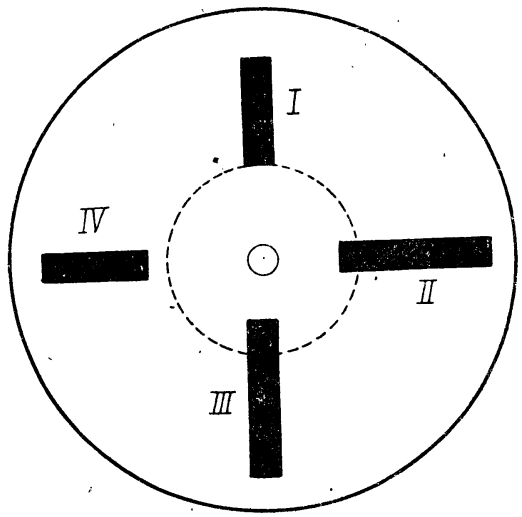

I. E. coli

II. Streptomycin-fast strain of E. coli

III. B. anthracis

IV. B. subtilis N. R. R.L. B-588

Fig. 2. Inhibition zones around colony producing streptomycin or streptothricin-group substances

\section{Isolation of streptomycin-producing strain}

An important publication by Waksman and others (4) shcwing the specific resistance of streptomycin-producing strain against streptomycin and the different characters of streptomycin from other substances resembling it found by us facilitated the isolation of streptomycin-producing strain. According to Waksman's description, one gram samples of soil is plated out, in dilutions of $1: 10^{4}, 1: 10^{5}, 1: 10^{7}$, with nutrient agar containing streptomycin at about $50 \mathrm{u} / \mathrm{cc}$. After incubation for 3-5 days actinomyces colonies growing are picked up and implanted in a spot on the nutrient agar plate ( $\mathrm{pH} 8.0$ ), and after 4 days at $28^{\circ} \mathrm{C}$ suspension of $\mathrm{E}$. coli is sprayed on the plate and the plate is incubated at $37^{\circ} \mathrm{C}$ for about 20 hours. Then actinomyces colonies showing the inhibition 
zones are picked up, implanted again in a spot on the nutrient agar plate ( $\mathrm{pH} 8.0$ ), and the plate is incubated for 4 days at $28^{\circ} \mathrm{C}$, and the streptomycin-fast strain and the normal strain of E. coli, B. subtilis N. R. R. L. B-558 and B. anthracis are'streaked radially from the colonies, and the plate is incubated for about 20 hours at $37^{\circ} \mathrm{C}$. If the colonies are producing streptomycin, then, as seen in Fig. 2, the normal culture of E. coli is much more strongly inhibited than the streptomycin-fast strain, and the inhibition zone of $\mathrm{B}$. anthracis is larger than that of B. subtilis. In the case of streptothricin-group substances, the relation of the inhibition zones of these four bacteria is much different from that of streptomycin.

The colony showing the production of streptomycin is isolated on slants of glucose asparagine agar, and afterwards its production of streptomycin is tested by shaking culture, putting $50 \mathrm{cc}$ of the culture medium ( $1 \%$ glucose, $0.5 \%$ beef or fish extract, $0.5 \%$ peptone, $0.5 \% \mathrm{NaCl}, 0.35 \% \mathrm{CaCO}_{3}, \mathrm{pH} 6.8-7.0$ ) into $350 \mathrm{cc}$ flask and shaking for 4 days at $25-30^{\circ} \mathrm{C}$. If the potency is to be tested by broth serial dilution method, sodium chloride must be removed from the testing broth, as it hinders the inhibiting effect of streptomycin. Often the freshly isolated strain of S. griseus does not produce the antibiotic substance in the first trial of shaking culture. Then it is plated out with nutrient agar plus streptomycin $(50 \mathrm{u} / \mathrm{cc})$ and afterwards several colonies are picked up and they are tested for the streptomycin production again by shaking culture.

.The strains which seem to produce streptomycin on the basis of their bacteriostatic

Table 3. The course of the isolation of S. griseus producing streptomycin

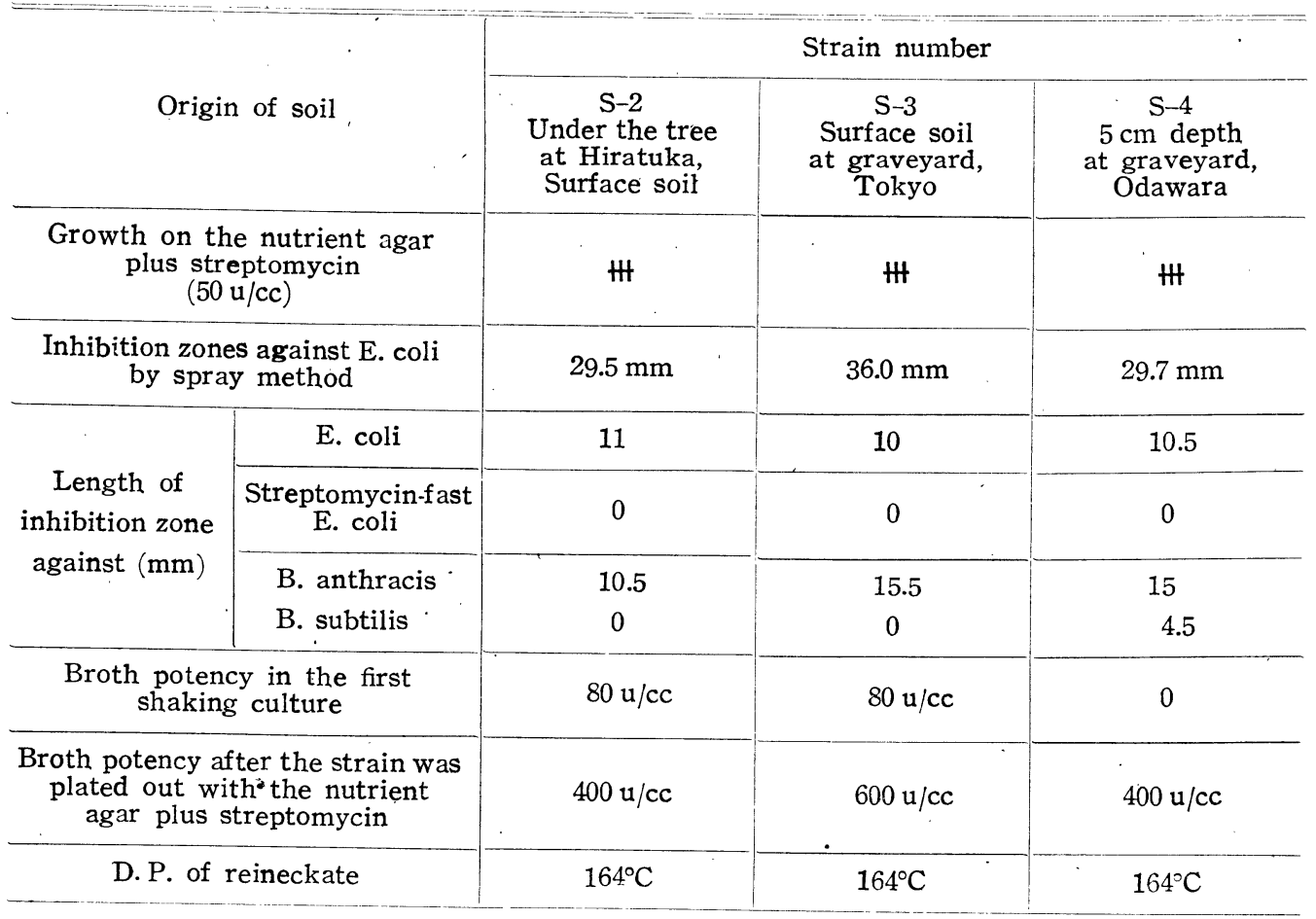


spectra are cultivated and from the broth streptomycin must be purified as its reineckate (D. P. $164^{\circ} \mathrm{C}$ ). In Table 3 , the courses during the isolation of three streptomycin-producing strains are described.

\section{Discussion}

According to Waksman (4) and others, Kelner and Morton (5), and Foster and others, (6) there seem to be many kinds of substances resembling streptomycin and streptothricin, but streptomycin is particular in respect of the specific tolerance of streptomycin-fast bacteria to. streptomycin and the bacteriostatic spectrum. These substances should be divided to streptomycin and other substances which we should like to call streptothricingroup substances.

Now it may be an important problem to find out how many kinds of substances are in the streptothricin-group. Here we should like to notice that the authors reporting various kinds of streptothricin-group substances differentiated these substances on the basis of antibacterial spectrum tested by serial broth dilution method. In our experience the differences of the spectrum appeard more markedly in the case of dilution method than when they were tested by the comparison of the width of inhibition zones on the agar plate. Besides it must be noticed, that almost all experiments on the streptothricingroup substances were performed by using impure materials. We think that the classification of streptothricin-group substances should be decided in future, particularly by the experiments on the purified samples. In this paper only seven strains of actinomyces producing streptothricin-group substances were studied, but further by testing the bacteriostatic activity against $\mathrm{E}$. coli, streptomycin-fast strain, streptothricin-group-fast strain, B. anthracis and B. subtilis, several strains of actinomyces such as A. fradii, A. sporeus, A. olivaceus, A. poolensis, etc., were also decided as the streptothricin-group. substance producing strains. So the streptothricin-group substances seem to be widely distributed among actinomyces.

Streptomycin could be differentiated by testing the tolerance of a streptomycin-fast bacteria to streptothricin-group substances. According to Reynolds and Waksman (7), grisein could be also differentiated from streptomycin by this method, so the method deciding the identity of streptomycin-producing strain described here should not be confused with the grisein-producing strain.

After we finished this work, we read the splendid work by Waksman, Reilly and Harris (8) concerning a rapid method for demonstrating the identity of streptomycinproducing strains. They utilized the specific actinophage to decide the identity of streptomycin-producing strain. Since specific actinophage is indispensable for this method it cannot be immediately adopted in our country.

\section{SUMMARY}

1) Streptmycin was different from other substances resembling it in the following points and we should like to call the latter the streptothricin-group substances. 
i) A streptomycin-fast strain of E. coli is much more resistant to streptomycin than the normal culture, but almost as susceptible to other substances.

ii) The relations of the resistances of B. subtilis N. R. R. L. B. -558 and B. anthracis against streptomycin and other substances are different.

iii) The Sakaguchi reaction showed beautiful red colour in the case of streptomycin hydrochloride, but brownish red colour in the case of streptothricin-group substances.

iv) The lately appeared toxicity could not be observed in the case of streptomycin.

2) A rapid method for demonstrating the identity of streptomycin-producing strain was described.

\section{REFERENCES}

1) Umezawa, H., Hayano, S., and Takeuchi, T.: Studies on the antibiotic substance from actinomyces. II. a basic antibiotic substance from several strains of actinomyces. Journal of Penicillin (Japan), 1, 138, 1947.

2) Umezawa, H., Dang Van Ngu, Takeuchi, T., and Sekizawa,.Y.: Studies on the antibiotic substance produced by actinomyces. Il. A slightly toxic and basic antibiotic substance from a strain of actinomyces. Journal of Penicillin (Japan), 1, 233, 1947.

3) Waksman, S. A.: Microbial antagonisms and antibiotic substances. New York, Commonwealth Fund 1945.

4) Waksman, S. A., Reilly, D. B., and Johnstone, D. B.: Isolation of streptomycin-producing strains of S. griseus. J. Bact., 52, 393, 1946.

5) Kelner, A., and Morton, H. E.: Two antibiotics (Lavendulin and actinorubin) produced by actinomyces. I. Isolation and characteristics of organisms. J. Bact., 53, 695-704, 1947.

6) Foster, J. W., and others. J. Bact., 51, 363, 1946.

7) Reynolds, M., Schatz, A., and Waksman, S. A.: Grisein, a new antibiotic produced by a strain of S. griseus. Proc. Soc. Exp. Biol. and Med., 64, 50-54, 1947.

8) Waksman, S. A., Reilly, H. C., and Harris, D. A.: A rapid method for demostrating the identity of streptomycin producing strains of S. griseus. Proc. Exp. Biol. Med., 66, 617, 1947. 